\title{
Calidad de vida y niveles de actividad física en el personal administrativo de universidades andinas
}

(c) (1)(3) (2)

Quality of life and levels of physical activity in the administrative staff of andean universitie

Edda Lorenzo Bertheau. ${ }^{1}$, Fausto Vinicio Sandoval Guampe. ${ }^{2}$, Isaac German Pérez Vargas. ${ }^{3}$, Bertha Susana Paz Viteri . ${ }^{4}$

\begin{abstract}
. DOI: https://doi.org/10.33262/cienciadigital.v3i2.5.534

The main objective is to determine the levels of physical activity and quality of life for the administrative staff of ANDEAN Universities. This research is descriptive, correlational, non-experimental cross-section with mixed methodology. The sample consisted of 200 people (111) Women and (89) Men, aged between 20 and 59 years, being the average of $(37.67 \pm 8.82)$ in women. The body mass index (BMI) obtained was DM=25.27 \pm 3.11 . To measure the quality of life level, the validated CVP-35 test was used and the IPAQ was used to determine the level of physical activity. It began with the application for the corresponding

\footnotetext{
${ }^{1}$ Universidad Nacional de Chimborazo, Facultad de Ciencias de la Educación, Humanas y Tecnologías, Carrera de Pedagogía de la Actividad Física y Deporte, Riobamba, Ecuador. elorenzo@unach.edu.ec

${ }^{2}$ Universidad Nacional de Chimborazo, Facultad de Ciencias de la Educación, Humanas y Tecnologías, Carrera de Pedagogía de la Actividad Física y Deporte, Riobamba, Ecuador. fsandoval@unach.edu.ec

${ }^{3}$ Universidad Nacional de Chimborazo, Facultad de Ciencias de la Educación, Humanas y Tecnologías, Carrera de Pedagogía de la Actividad Física y Deporte, Riobamba, Ecuador. iperez@unach.edu.ec

${ }^{4}$ Universidad Nacional de Chimborazo, Facultad de Ciencias de la Educación Humanas y Tecnologías, Carrera de Pedagogía de la Actividad Física y Deporte, Riobamba, Ecuador.spaz@unach.edu.ec
} 
permits to work with the administrative staff of the different universities. Once counting on them they proceeded to explain the purpose of the research and that they will be under the conditions of anonymity, requesting written consent. Finally, the working protocol was applied. The quality of life is subdivided into three dimensions, managerial support, workload and intrinsic motivation. Obtaining an average of $7.05 \pm 2.33$ in the Management Support scale, an average of $6.03 \pm 2.28$ in the sub-scale of workload, and an average of $7.66 \pm 2,07$ in the intrinsic motivation scale. Instrument with a high degree of reliability with Cronbach's Alpha (0.85). The physical activity levels of the study population were measured in metabolic expenditure, resulting in 708 mets. A correlation between the variables was applied, giving a significant relationship between several sub-scales. It is concluded that the administrative personnel of the Andean universities consider that they have enough managerial support, a lot of workload and have enough intrinsic motivation. Physical activity levels were moderate and there is a relationship between managerial support - intrinsic motivation; managerial support - metabolic expenditure and finally between intrinsic motivation - metabolic expenditure.

\section{Key Words: physical inactivity, metabolic expense}

\section{Resumen.}

El objetivo general es determinar los niveles de actividad física y la calidad de vida en el personal administrativo de Universidades ANDINAS. Esta investigación es descriptiva, correlacional, no experimental de corte transversal con metodología mixta. La muestra conformada por 200 personas (111) Mujeres y (89) Hombres, de edades comprendidas entre 20 y 59 años, siendo la media en mujeres $(37,67 \pm 8,82)$. El Índice de masa corporal (IMC) obteniendo fue $\mathrm{DM}=25,27 \pm 3,11$. Para medir el nivel de calidad de vida se utilizó el test validado CVP- 35 y para determinar el nivel de actividad física se utilizó el IPAQ. Se inició con la solicitud de los permisos correspondientes para trabajar con el personal administrativo de las diferentes universidades. Una vez contando con ellos se procedió a explicar el objetivo del trabajo y las condiciones de anonimato, solicitándoles el consentimiento escrito. Por último, se procedió a la aplicación del protocolo de trabajo. La calidad de vida se subdivide 
en tres dimensiones, Apoyo directivo, Carga de trabajo y Motivación intrínseca. Obteniéndose en la escala Apoyo directivo una media de 7,05 $\pm 2,33$, en la sub-escala carga de trabajo una media de 6,03 $\pm 2,28$, y en la escala motivación intrínseca una media de $7,66 \pm 2,07$. Instrumento con alto grado de fiabilidad con Alfa de Cronbach $(0,85)$. Los niveles de actividad física de la población de estudio se midieron en gasto metabólico, dando como resultado 708 mets. Se aplicó una correlación entre las variables dando una relación significativa entre varias sub-escalas. Se concluye que el personal administrativo de las universidades andinas considera que tienen bastante apoyo directivo, bastante carga de trabajo y tienen bastante motivación intrínseca. Los niveles de actividad física fueron moderados y existen una relación entre el apoyo directivo - motivación intrínseca; apoyo directivo - gasto metabólico y por último entre la motivación intrínseca - el gasto metabólico.

Palabras claves: Inactividad Física, Gasto Metabólico

\section{Introducción.}

La inactividad física constituye el cuarto factor de riesgo más importante de mortalidad en todo el mundo (6\% de defunciones a nivel mundial). Sólo la superan la hipertensión (13\%), el consumo de tabaco (9\%) y el exceso de glucosa en la sangre (6\%). El sobrepeso y la obesidad representan un 5\% de la mortalidad mundial (World Health Organization, 2018).

En una investigación realizada en la población española el 6\% de los españoles que practican deporte lo hacen en 'sus puestos de trabajo o como actividad en el centro de enseñanza'. Por el contrario, el 68\% de los españoles practica deporte 'por su cuenta' y el 24\% lo hace 'como actividad de un club, asociación, organización o federación'. Este resultado se mantiene estable o desciende ligeramente, con respecto al mismo resultado del estudio de características similares realizado por (Barranco-Ruiz, 2017).

Al menos el $60 \%$ de la población mundial no realiza suficiente actividad física como para obtener beneficios para la salud, estos datos se incrementan en algunos países, como en Chile, donde el sedentarismo aumenta en todos los grupos de edades, a cifras que alcanzan hasta el $91 \%$, por lo que constituyen hoy en día un problema epidemiológico que va más allá de las 
barreras socioculturales, debido, en gran parte, al incremento de transporte y de los índices de urbanización asociado a un crecimiento económico y social; esta información contrasta con anteriores encuestas donde el sedentarismo tiene una mayor prevalencia en los niveles socioeconómicos bajos, en las personas con menor nivel de escolaridad y más alto en las mujeres que en los hombres (Malo-Serrano, 2017) como ocurre ocasionalmente en el deporte competitivo y en algunas actividades laborales pesadas. De otro lado, se vienen estudiando el riesgo que representa para una persona o para una comunidad, la falta de actividades musculares vigorosas y regulares, situación ésta cada vez más frecuente, en la medida en que el desarrollo tecnológico releva a las personas de toda clase de esfuerzo físico (Serrano, 2018).

En el Ecuador lamentablemente no ha existido una política que permita promover programas de actividad física en el ambiente laboral. Sin embargo, el Ministerio del Deporte con el objetivo de disminuir el sedentarismo y reducir el riesgo de enfermedades ocasionadas por el esfuerzo intelectual y la poca actividad corporal, desde el mes de noviembre reinició en Esmeraldas el proyecto denominado "PAUSA ACTIVA LABORAL", que consiste en una rutina de estiramientos y ejercicios realizados por funcionarios de distintas instituciones durante su jornada laboral durante 15 minutos diarios (Ministerio del Deporte, 2012).

\section{Metodologia.}

\subsection{PARTICIPANTES}

La muestra de este estudio estará formada por 200 (111 mujeres, 89 Hombres) empleados administrativos en el periodo abril - agosto del año 2018 de universidades ANDINAS.

\subsection{DISEÑO}

Esta investigación es descriptiva, correlacional, no experimental de corte transversal. Con una metodología mixta (cualitativa y cuantitativa).

\subsection{PROCEDIMIENTO}

El proceso se inició con la solicitud a la Escuela Superior Politécnica de Chimborazo de los permisos correspondientes para poder trabajar con los empleados administrativos. Una vez 
que se contó los permisos pertinentes se procedió a explicará a los administrativos el objetivo del trabajo y las condiciones de anonimato. Una vez Contestado el cuestionario de preguntas se les solicito el consentimiento escrito. Posteriormente se procedió a la aplicación del protocolo de trabajo.

Una vez obtenidos los resultados de las encuestas se realizó la tabulación de datos utilizando el programa Excel e IBM SPSS “Paquete Estadístico para las Ciencias Sociales” lo que permitió realizar el análisis de los resultados de las dos variables de estudio.

\subsection{VARIABLES DE ESTUDIO}

2.4.1. La variable Independiente calidad de vida se medirá con el cuestionario CVP-35 por (Martín, y otros, 2004), el cual consta de 35 ítems que se responden en una escala de 1 a 10, a la que se superponen las categorías. Valora 3 dimensiones: Apoyo directivo (AD), Cargas en el trabajo (CT) y Motivación Intrínseca (MI).

- $\quad$ nada (valores 1 y 2)

- $\quad$ algo (valores 3, 4 y 5)

- $\quad$ bastante (valores 6,7 y 8 )

- $\quad$ mucho (valores 9 y 10)

2.4.2. La variable Dependiente. Niveles de Actividad Física se medirá con la Escala International physical activity questionnaire (IPAQ) el cual sirve para monitorear el nivel de actividad física en una población para uso con jóvenes y adultos de mediana edad entre (1569 años) y posteriores algunos trabajos y comprobaciones no recomiendan su uso con edades mayores o menores. Hay tres niveles de actividad física propuestas para clasificar poblaciones:

- $\quad$ Baja: No registran actividad física o la registra, pero no alcanza las categorías media y alta.

- Moderada: Considera los siguientes criterios:

> 3 o más días de actividad física vigorosa por lo menos 20 min por día.

$>\quad 5$ o más días de actividad física de intensidad moderada o caminar por lo menos 30 min. 
5 o más días de cualquier combinación de actividad física leve, moderada o vigorosa que alcancen un registro de $600 \mathrm{METs}-\mathrm{min} / \mathrm{semana}$.

- Alta: Es una categoría alta y cumple los siguientes requerimientos:

3 o más días de actividad física vigorosa o que acumulen 1.500 METs-minsemana.

7 o más días de cualquier combinación de actividad física leve, moderada o vigorosa que alcance un registro de $3.000 \mathrm{METs}-\mathrm{min} / \mathrm{semana}$.

Los valores del MET seccionados se derivan del trabajo realizado durante los estudios de fiabilidad de IPAQ acometidos en 2000-2001. Usando el Compendio de (Mocha-Bonilla Julio, A., Alava, D. J. H., Muñoz, J. M. V., \& Castro, N. J. V, 2018) podemos extraer unos METs promedio como resultado de cada tipo de actividad. Por ejemplo; se incluyen todos los tipos de "andar y se crea un valor medio de METs para andar". El mismo procedimiento se ha tomado para las actividades de intensidad moderada y las actividades de intensidad vigorosa. Los siguientes valores se usan para el análisis de los resultados de IPAQ: Andar $=3.3 \mathrm{METs}$ y AF vigorosa $=8.0$ METs. Usando estos valores, se definen cuatro resultados continuos.

\section{Resultados.}

Análisis del cuestionario Calidad de vida "CVP-35" del personal administrativo de universidades ANDINAS.

Se realizó la evaluación del nivel de Calidad de vida a los empleados administrativos con una población de estudio de 200 personas, 111 mujeres $(55,5 \%)$ y 89 Hombres equivalente a (44,5\%), que oscila en edades entre (20-59 años), siendo la media en mujeres de (37,67士 $8,82)$ y en los hombres de $(36,89 \pm 8,74)$.

En comparación con el estudio realizado por (Morales, A., \& Yanina, E., 2018)en la Universidad de San Martin Perú, con una muestra intencional conformada por 198 participantes, de los cuales el $72,2 \%$ eran mujeres y el $26,8 \%$ hombres se puedo determinar que la población de estudio que predomina es el género femenino en ambos casos, al igual que nuestro estudio. 
Dentro de la evaluación del cuestionario CVP-35 del estudio realizado además del genero también se consideró las siguientes variables: edad, estatura, peso e Índice de masa corporal "IMC".

\subsection{Análisis de las variables IMC.}

La estatura registro un promedio $\mathrm{DM}=1,58 \mathrm{~m} \pm 0,06$., el peso obtuvo como resultado $\mathrm{DM}=$ $60,88 \mathrm{~kg} \pm 7,16$. El índice de masa corporal de la muestra de estudio femenina fue de $\mathrm{DM}=$ $24,32 \pm 2,71$ y de la muestra masculina fue de $\mathrm{DM}=25,27 \pm 3,11$. Se determinó que el IMC en donde se registrar un promedio más alto en los hombres respecto a las mujeres.

\subsection{Análisis de los resultados de la variable Calidad de vida Profesional}

Para el análisis de la encuesta CVP-35 se dividió en tres grupos:

a) Apoyo Directivo la cual consta de las preguntas: (P2- P3- P4- P5- P10- P11- P14- P16P20- P22- P23- P28- P30) su contenido y equivalente se puede observar en la tabla pág. $\mathrm{N}^{0}$ 20. Obteniendo como resultado el promedio más alto en la $\mathrm{P2}$ con un promedio $\mathrm{DM}=\mathbf{8 , 5 8} \pm \mathbf{1 , 5 0}$ el resultado más bajo se puede observar en $\mathrm{P4}$ con un promedio $\mathrm{MD}=\mathbf{5 , 8 9} \pm 2,72$ resultado que se puede observar en la tabla $\mathrm{N}^{\circ} 1$.

Tabla Na 1 Análisis resultados Apoyo directivo

\begin{tabular}{|c|c|c|c|c|c|c|c|c|c|c|c|c|c|}
\hline \multicolumn{14}{|c|}{ Estadistico } \\
\hline & P2 & P3 & P4 & P5 & P10 & P11 & P14 & P16 & P20 & P22 & P23 & P28 & P30 \\
\hline Válido & 200 & 200 & 200 & 200 & 200 & 200 & 200 & 200 & 200 & 200 & 200 & 200 & 200 \\
\hline Perdidos & 0 & 0 & 0 & 0 & 0 & 0 & 0 & 0 & 0 & 0 & 0 & 0 & 0 \\
\hline Media & $8,58 \pm 1,50$ & $6,82 \pm 2,37$ & $5,89 \pm 2,72$ & $6,68 \pm 2,46$ & $7,87 \pm 2,24$ & $7,96 \pm 2,02$ & $8,09 \pm 1,87$ & $6,43 \pm 3,05$ & $6,48 \pm 2,59$ & $6,23 \pm 2,56$ & $6,61 \pm 2,31$ & $7,06 \pm 2,24$ & $7,01 \pm 2,35$ \\
\hline
\end{tabular}

Fuente: Elaboración Propia

En la tabla $\mathrm{N}^{\circ} 1$ se puede observar el análisis de la dimensión Apoyo Directivo en la cual registra el promedio más alto en la pregunta número $2 \mathrm{DM}=8,58 \pm 1,50$ equivalente a "Mucho" lo que permite determinar que la población de estudio está muy conforme con la jornada 
laboral. El promedio más bajo se obtuvo de la pregunta número $4 \mathrm{DM}=5,89 \pm 2,72$ equivalente a "Bastante" determinando que existe posibilidades de ascender a un cargo más alto en la dependencia laboral a la que pertenece, obteniendo un promedio global $\mathrm{DM}=7,05 \pm 2,33$ en esta dimensión.

Se aplicó el alfa de Cronbach para la sub-escala presentando una consistencia interna alta 0 , 86 posteriormente se aplicó una ANOVA de Friedman la cual mostro un nivel de significación $(p=0,000)$ confirmando los resultados encontrados. En estudios similares realizado por (Martín Fernández, y otros, 2008) no coinciden con nuestros resultados ya que sus datos resultaron ser bajos con un valor $\mathrm{DM}=4.59$.

En comparación con el estudio realizado por (García-Sanz, E., Jiménez-Rejano, J. J., PérezCabezas, V., Chillón-Martínez, R., de la Casa Almeida, M., \& Rebollo-Salas, M., 2018) la cual registra un promedio $\mathrm{DM}=4,78$ equivalente a "Algo" en la dimensión apoyo directivo. Esto determina que en la investigación realizada los directivos de esta institución presta mayor atención y apoyo a sus trabajadores.

b) Cargas de Trabajo. - la cual consta de las preguntas (P1- P6- P7- P8- P17- P18- P19P21- P24-P25- P33) su definición y equivalente se puede observar en la tabla $\mathbf{N}^{\circ} 2$.

Tabla N 2 Análisis Cargas de Trabajo

\begin{tabular}{lccccccccccc}
\hline & P1 & P6 & P7 & P8 & P17 & P18 & P19 & P21 & P24 & P25s & P33 \\
Válido & 200 & 200 & 200 & 200 & 200 & 200 & 200 & 200 & 200 & 200 & 200 \\
Perdidos & 0 & 0 & 0 & 0 & 0 & 0 & 0 & 0 & 0 & 0 & 0 \\
Media & $8,425 \pm 1,42$ & $7,16 \pm 2,24$ & $7,24 \pm 2,23$ & $6,39 \pm 2,39$ & $3,21 \pm 2,32$ & $5,22 \pm 2,45$ & $4,58 \pm 2,69$ & $7,95 \pm 1,86$ & $4,82 \pm 2,47$ & $6,09 \pm 2,41$ & $5,3 \pm 2,65$ \\
\hline Media Global 6,03 22,28 \\
Fuente: Elaboración Propia
\end{tabular}

En la tabla $\mathrm{N}^{\mathrm{o}} 2$ se puede observar el análisis de la dimensión Cargas de trabajo obteniendo el promedio de la media más alta $8,43 \pm 1,42$ en la pregunta número 1 equivalente a "Bastante" lo que permite determinar que la población en estudio tiene bastante trabajo durante la 
jornada laboral, el promedio más bajo se registró en la pregunta número $17 \mathrm{DM}=3,21=2,32$ equivalente a "Algo" determinando que existe algo de conflicto entre los trabajadores, El estudio registro una media global para esta dimensión $\mathrm{DM}=6,03 \pm 2,28$ que significa bastante.

Se aplicó el alfa de Cronbach para la sub-escala presentando una consistencia interna alta 0 , 74, posteriormente se aplicó una ANOVA de Fridman la cual mostro un nivel de significación $(p=0,000)$ confirmando los resultados encontrados. En estudios similares realizado por (Moya, P., CARO, J. C., \& Monsalves, M. J., 2017) coinciden con nuestros resultados ya que sus datos resultaron ser bastante con un valor $\mathrm{DM}=4.59$.

En comparación con el estudio realizado por (García-Sanz, E., Jiménez-Rejano, J. J., PérezCabezas, V., Chillón-Martínez, R., de la Casa Almeida, M., \& Rebollo-Salas, M., 2018) la cual registra un promedio $\mathrm{DM}=6,03$ equivalente a "Bastante" en la dimensión cargas de trabajo. Determinando que en los dos estudios existe bastante carga de trabajo debido a que existe una similitud en la pregunta número 1 .

c) Motivación Intrínseca la cual consta de las preguntas: (P9- P12- P13- P15- P26- P27P29- P31- P32- P34- P35- P36) su definición y equivalente se puede observar en la tabla No3.

Tabla Na 3 Motivación Intrínseca

\begin{tabular}{lcccccccccccc}
\hline & & & & & & & & & & & \\
& P9 & P12 & P13 & P15 & P26 & P27 & P29 & P31 & P32 & P34 & P35 & P36 \\
Válido & 200 & 200 & 200 & 200 & 200 & 200 & 200 & 200 & 200 & 200 & 200 & 200 \\
Perdidos & 0 & 0 & 0 & 0 & 0 & 0 & 0 & 0 & 0 & 0 & 0 & 0 \\
Media & $7,75 \pm 2,34$ & $8,9 \pm 1,62$ & $8,86 \pm 1,38$ & $6,4 \pm 2,52$ & $5,73 \pm 2,77$ & $8,11 \pm 1,98$ & $8,14 \pm 2,14$ & $8,05 \pm 1,79$ & $8,67 \pm 1,74$ & $7,38 \pm 2,07$ & $7,43 \pm 2,11$ & $6,46 \pm 2,36$ \\
\hline Media global $7,66 \pm 2,07$
\end{tabular}

Fuente: Elaboración Propia

En la tabla $\mathrm{N}^{0} 3$ se puede observar el análisis de la dimensión motivación intrínseca obteniendo el promedio de la media más alta $\mathrm{DM}=8,90 \pm 1,62$ en la pregunta número 12 equivalente a "Mucho" lo que permite determinar que la población en estudio tiene un apoyo incondicional de la familia, el promedio más bajo se registró en la pregunta número 17 $\mathrm{DM}=5,73 \pm 2,77$ equivalente a "Bastante" determinando que existe interés de la Escuela 
Superior Politécnica de Chimborazo capacitar constantemente a sus trabajadores. El estudio registro una media global para esta dimensión $\mathrm{DM}=7,66 \pm 2,07$ que significa bastante.

Se aplicó el alfa de Cronbach para la sub-escala presentando una consistencia interna alta 0 , 70, posteriormente se aplicó una ANOVA de Fridman la cual mostro un nivel de significación $(\mathrm{p}=0,000)$ confirmando los resultados encontrados. En estudios similares realizado por (Moya, P., CARO, J. C., \& Monsalves, M. J., 2017) coinciden con nuestros resultados ya que sus datos resultaron ser bajos con un valor promedio $\mathrm{DM}=6,22$.

En comparación con el estudio realizado por (Morales, A., \& Yanina, E., 2018) con relación a la dimensión motivación intrínseca presenta un rango de una buena calidad de vida determinando un similar resultado con el estudio realizado en la Escuela Superior Politécnica de Chimborazo.

Se analizó la validez de la encuesta CVP- 35, para lo cual se calculó alfa de Cronbach utilizándose el programa SPSS y los 36 ítems de la encuesta obtenido como resultado $\alpha$ Cronbrach $=0,85$ lo que determina que la investigación realizada en la Escuela Superior Politécnica de Chimborazo es de buena confiabilidad ya que el valor de alfa cuando más se entra cerca de 1 es mayor la consistencia (Namdeo, S. K., \& Rout, S. D., 2017).

En comparación con el estudio realizado por Grimaldo Miriam, 2010 con un equivalente $\alpha$ de Cronbach $=0,76$ se registra una diferencia de 0,09 determinando que existe mayor confiabilidad y fiabilidad en el estudio realizado en la Escuela Superior Politécnica de Chimborazo.

cuestionario Internacional de Actividad Física "IPAQ" del personal administrativo de la Escuela Superior Politécnica de Chimborazo.

Una vez terminada la recolección de los datos se procedió al análisis utilizando el programa Excel para el cálculo de los METs y las Kcal de todas las personas encuestadas obteniendo la totalidad de los METs/min/semana. Esto permitió analizar en el programa SPSS la Media y Moda resultados que se pueden observar en la tabla $\mathrm{N}^{\circ} 4$ 
Tabla Na 4 Análisis de los METs

\begin{tabular}{|c|c|c|c|c|c|}
\hline \multicolumn{6}{|c|}{ Estadísticos descriptivos } \\
\hline TOTAL & $\mathrm{N}$ & Mínimo & Máximo & Media & Desviación estándar \\
\hline $\mathrm{MET} / \mathrm{min} / \mathrm{W}$ & & & & & \\
\hline EEK_PRE/3 & 200 & 0,00 & 96232 & 4959,74 & 8853,63 \\
\hline
\end{tabular}

Fuente: Elaboración Propia

Como se puede observar en la tabla $\mathrm{N}^{0} 4$ el análisis realizado de la totalidad de los $\mathrm{METs} / \mathrm{min} / \mathrm{semana}$ determinando la media de la muestra del estudio $\mathrm{DM}=4959,74 \pm 8853,63$ con un mínimo de cero y un máximo de 96232METs/min/semanas. Después de computarizar los METs/min/semanas $(4959,74$ / 7 (días de la semana) =708 mets, lo que demuestra que el personal administrativo se encuentra con una condición física Moderada. En estudios realizados en población chilena, aplicando el mismo instrumento de evaluación, los resultados mostraron conclusiones similares (niveles bajos y moderados de actividad física), aunque nosotros no diferenciamos por género, ni edad (Morales, G., Balboa-Castillo, T., Muñoz, S., Belmar, C., Soto, Á., Schifferli, I., \& Guillen-Grima, F., 2017).

Para analizar la correlación entre las variables de estudio se aplicó la correlación bivariadas, las cuales muestran como resultado que existe una relación entre el apoyo directivo y las motivaciones intríncaselas $(\mathrm{p}=0,000)$. También muestra una relación significativa entre el apoyo directivo y el gasto metabólico $(\mathrm{p}=0,003)$. Por otro lado, se observa una relación significativa entre la motivación intrínseca y el gasto metabólico, ver tabla N. 5.

Tabla 5 - Correlación Bivariada entre todas las variables.

\begin{tabular}{llcccc} 
& & AD & CT & MI & mets \\
\hline AD & Correlación de Pearson & 1 & 0,076 &, $671^{* *}$ &, $210^{* *}$ \\
& Sig. (bilateral) & & 0,287 & 0 & 0,003 \\
\hline CT & Correlación de Pearson & 0,076 & 1 & 0,087 &,$- 175^{*}$ \\
& Sig. (bilateral) & 0,287 & & 0,221 & 0,013 \\
\hline \multirow{2}{*}{ MI } & Correlación de Pearson &, $671^{* *}$ & 0,087 & 1 &, $232^{* *}$ \\
& Sig. (bilateral) & 0 & 0,221 & & 0,001 \\
\hline \multirow{2}{*}{ mets } & Correlación de Pearson &, $210^{* *}$ &,$- 175^{*}$ &, $232^{* *}$ & 1 \\
& Sig. (bilateral) & 0,003 & 0,013 & 0,001 & \\
\hline
\end{tabular}


**. La correlación es significativa en el nivel 0,01 ( 2 colas).

*. La correlación es significativa en el nivel 0,05 ( 2 colas).

Fuente: Elaboración Propia

\section{Conclusiones.}

1. Los resultados revelan que el nivel de actividad física en el personal administrativo de las Universidades ANDINAS que participo en la investigación fue de Moderado.

2. Al evaluar la calidad de vida se tomó en consideración los 3 aspectos, determinándose el personal administrativo está satisfecho con el horario de trabajo establecido, que tiene bastante trabajo durante la jornada laboral y que el apoyo familiar es muy importante en el desarrollo de su trabajo.

3. Al correlacionar las variables niveles de actividad física y calidad de vida profesional se determina que existe una relación entre apoyo directivo y motivación intrínseca, apoyo directivo con el gasto metabólico y motivación intrínseca con el gasto metabólico.

4. Implementar un programa de pausa activa durante la jornada laboral, según la Organización Mundial de la Salud (OMS) que permita mantener e incrementar el nivel de actividad fisca y mejorar la calidad de vida de sus trabajadores. Sensibilizar a los directivos sobre la importancia de la motivación intrínseca para el desempeño laboral y gasto metabólico. Fomentar actividades deportivas en equipo los días viernes, para fortalecer la cohesión grupal.

\section{Referencias bibliográficas.}

Aldana, S., Vereda, F., Hidalgo-Alvarez, R., \& de Vicente, J. (2016). Facile synthesis of magnetic agarose microfibers by directed selfassembly. Polymer, 93, 61-64.

Barranco-Ruiz, Y. M.-A.-V. (2017). Una práctica de entrenamiento competitivo de por vida atenúa la peroxidación lipídica relacionada con la edad. Revista de fisiología y bioquímica. 
Bhat, S., Tripathi, A., \& Kumar, A. (2010). Supermacroprous chitosan-agarose-gelatin cryogels. in vitro characterization and in vivo assesment for cartilage tissue engineering. Journal of the Royal Society Interface, 1-15.

Bossis, G., Marins, J., Kuzhir, P., Volkova, O., \& Zubarev, A. (2015). Functionalized microfibers for field-responsive materials and biological applications. Journal of Intelligent Material Systems and Structures, 1-9.

Cortés, J., Puig, J., Morales , J., \& Mendizábal, E. (2011). Hidrogeles nanoestructurados termosensibles sintetizados mediante polimerización en microemulsión inversa. Revista Mexicana de Ingeniería Química., 10(3), 513-520.

Dias, A., Hussain, A., Marcos, A., \& Roque, A. (2011). A biotechnological perspective on the application of iron oxide magnetic colloids modified with polysaccharides. Biotechnology Advances 29, 29, 142-155.

Estrada Guerrero, R., Lemus Torres, D., Mendoza Anaya, D., \& Rodriguez Lugo, V. (2010). Hidrogeles poliméricos potencialmente aplicables en Agricultura. Revista Iberoamericana de Polímeros, 12(2), 76-87.

García-Cerda, L., Rodríguez-Fernández, O., Betancourt-Galindo, R., Saldívar-Guerrero, R., \& TorresTorres, M. (2003). Síntesis y propiedades de ferrofluidos de magnetita. Superficies y Vacío., 16(1), 28-31.

García-Sanz, E., Jiménez-Rejano, J. J., Pérez-Cabezas, V., Chillón-Martínez, R., de la Casa Almeida, M., \& Rebollo-Salas, M. (2018). Calidad de vida profesional de los fisioterapeutas en el ámbito hospitalario público de Sevilla. Dialnet, 65-72.

Ilg, P. (2013). Stimuli-responsive hydrogels cross-linked by magnetic nanoparticles. Soft Matter, 9, 3465-3468.

Lewitus, D., Branch, J., Smith, K., Callegari, G., Kohn, J., \& Neimark, A. (2011). Biohybrid carbon nanotube/agarose fibers for neural tissue engineering. Advanced Functional Materials, 21, 2624-2632.

Lin, Y.-S., Huang, K.-S., Yang, C.-H., Wang, C.-Y., Yang, Y.-S., Hsu, H.-C., . . Tsai, C.-W. (2012). Microfluidic synthesis of microfibers for magnetic-responsive controlled drug release and cell culture. PLOS ONE, 7(3), 1-8.

Malo-Serrano, M. C. (2017). Active breack as a changing factor in the physical activity of public officials. 37, 303-313.

Martín Fernández, j., Gómez Gascón, T., Martínez García-Olalla, C., del Cura González, M., Cabezas Peña, M., \& García Sánchez, S. (july de 2008). Medición de la capacidad evaluadora del cuestionario CVP-35 para la percepción de la calidad de vida profesional. (ELSEVIER, Ed.) Atención Primaria, 40(7), 327-334. doi:https://doi.org/10.1157/13124124 
Martín, J., Alfonso Cortés, J., Morente, M., Caboblanco, M., Garijo, J., \& Rodríguez, A. (mar-abr de 2004). Características métricas del Cuestionario de Calidad de Vida Profesional (CVP-35) . Gaceta Sanitaria, 18(2).

Ministerio del Deporte. (2012). Pausa Activa Laboral.

Mocha-Bonilla Julio, A., Alava, D. J. H., Muñoz, J. M. V., \& Castro, N. J. V. (2018). OBESITY AND SEDENTARISM LEVELS ANALYSIS: A CASE STUDY UNIVERSIDAD TÉCNICA DE AMBATOINGAHURCO CAMPUS. International Journal of Sciences and Research, 74 (5/1). doi:10.21506

Morales, A., \& Yanina, E. (marzo de 2018). CALIDAD DE VIDA PROFESIONAL EN ESTUDIANTES DE CIENCIAS DE LA SALUD. Revista de Psicología, 12(1).

Morales, G., Balboa-Castillo, T., Muñoz, S., Belmar, C., Soto, Á., Schifferli, I., \& Guillen-Grima, F. (2017). sociación entre factores de riesgo cardiometabólicos, actividad física y sedentarismo en universitarios chilenos. Nutrición Hospitalaria, 1345-1352. Obtenido de http://scielo.isciii.es/pdf/nh/v34n6/14_morales.pdf

Moya, P., CARO, J. C., \& Monsalves, M. J. (2017). Percepción de la Calidad de Vida Profesional de docentes de Odontología. Revista de Educación en Ciencias de la Salud, 14(2), 2. Obtenido de https://dialnet.unirioja.es/servlet/articulo?codigo=6343353

Namdeo, S. K., \& Rout, S. D. (2017). Calculating and interpreting Cronbach's alpha using Rosenberg assessment scale on paediatrician's attitude and perception on self esteem. International Journal of Community Medicine and Public Health, 1371-1374. Obtenido de https://scholar.google.com.ec/scholar?hl=es\&as_sdt=0\%2C5\&as_ylo=2017\&q=Calculating $\% 2 \mathrm{C}+$ Interpreting $\% 2 \mathrm{C}+\mathrm{And}+$ Reporting+Cronbach\%C2\%B4s+Alpha+Reliability+Coefficient $+\mathrm{f}$ or+Likert-Type+scales.\&btnG=

Ruiz Estrada, G. (2004). Desarrollo de un Sistema de liberación de fármacos basado en nanopartículas magnéticas recubiertas con Polietilénglicol para el tratamiento de diferentes enfermedades. Madrid: Universidad Autónoma de Madrid. Departamento de Física Aplicada.

Serrano, S. (Mayo de 2018). Inactividad Física y ausentismo en el Ámbito Laboral. Revista de Salud Pública, 10(2), 227-238.

Song , J., King, S., Yoon , S., Cho, D., \& Jeong, Y. (2014). Enhanced spinnability of narbon nanotube fibers by surfactant addition. Fiberes and Polymers, 15(4), 762-766.

Tartaj, P., Morales, M., González-Carreño, T., Veintemillas-Verdaguer, S., \& Serna, C. (2005). Advances in magnetic nanoparticles for biotechnology applications. Journal of Magnetism and Magnetic Materials, 290, 28-34. 
World Health Organization. (2018). Recomendaciones mundiales sobre actividad física para la salud. (pág. 58). Londres Reino Unido: Ediciones de la OMS. Obtenido de https://apps.who.int/iris/bitstream/handle/10665/44441/9789243599977_spa.pdf

Wulff-Pérez , M., Martín-Rodriguez, A., Gálvez-Ruiz, M., \& de Vicente, J. ( 2013 ). The effect of polymer surfactant on the rheological properties of nanoemulsions. Colloid and Polymer Science, 291, 709-716.

Zamora Mora, V., Soares, P., Echeverria, C., Hernández , R., \& Mijangos, C. (2015). Composite chitosan/Agarose ferrogels for potential applications in magnetic hyperethermia. Gels., 1 , 69-80.

\section{PARA CITAR EL ARTÍCULO INDEXADO.}

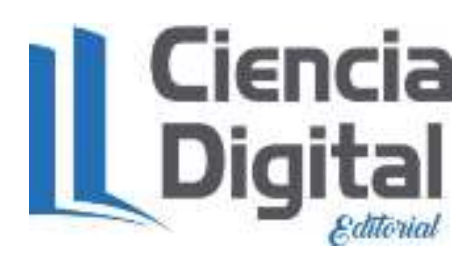

Lorenzo Bertheau, E., Sandoval Guampe, F., Pérez Vargas, I., \& Paz Viteri, B. (2019). Calidad de vida y niveles de actividad física en el personal administrativo de universidades andinas. Ciencia Digital, 3(2.5), 90-104. https://doi.org/10.33262/cienciadigital.v3i2.5.534
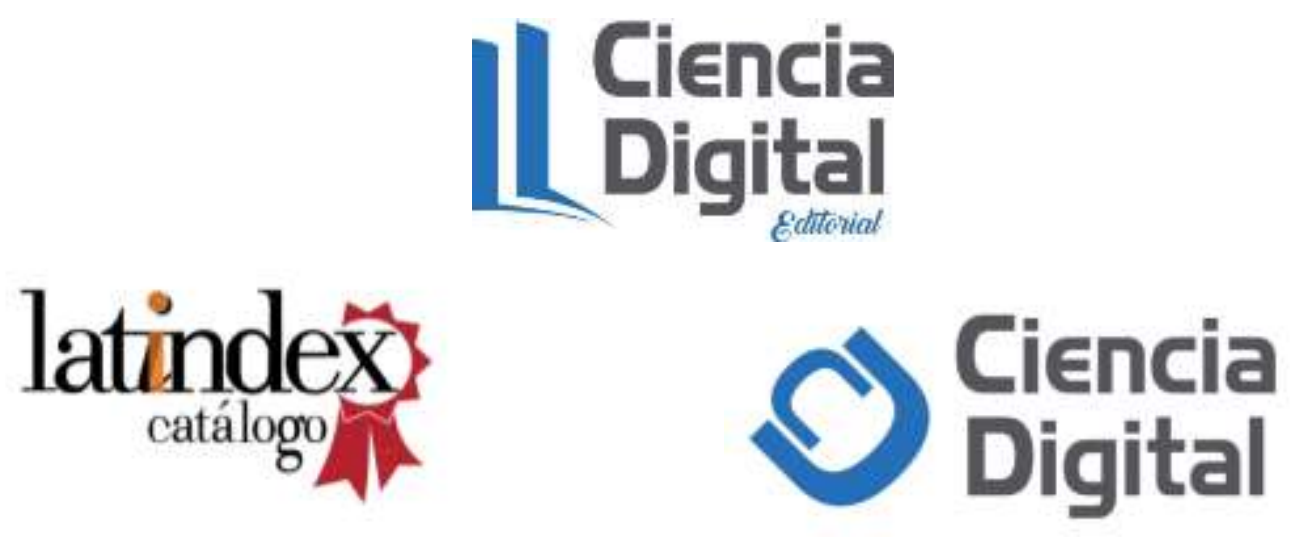